\title{
Enjeux et fractures de la Bolivie en 2009
}

Analyse des consultations référendaires du 25 janvier

\section{Sébastien Hardy}

\section{OpenEdition}

Journals

\section{Édition électronique}

URL : http://journals.openedition.org/echogeo/10965

DOI : $10.4000 /$ echogeo.10965

ISSN : 1963-1197

\section{Éditeur}

Pôle de recherche pour l'organisation et la diffusion de l'information géographique (CNRS UMR 8586)

Référence électronique

Sébastien Hardy, « Enjeux et fractures de la Bolivie en 2009 », EchoGéo [En ligne], Sur le Vif, mis en ligne le 09 mars 2009, consulté le 30 avril 2019. URL : http://journals.openedition.org/echogeo/10965 ; DOI : 10.4000/echogeo.10965

Ce document a été généré automatiquement le 30 avril 2019.

\section{(c) (i) (3)}

EchoGéo est mis à disposition selon les termes de la licence Creative Commons Attribution - Pas d'Utilisation Commerciale - Pas de Modification 4.0 International 


\section{Enjeux et fractures de la Bolivie en 2009}

Analyse des consultations référendaires du 25 janvier

\section{Sébastien Hardy}

\section{Une nouvelle Constitution, formule quasi magique de refondation de la Bolivie}

1 L'élection le 18 décembre 2005 d'Evo Morales Ayma à la présidence de la République a permis à la Bolivie d'entamer un processus de profond renouvellement, parfois même qualifié d'historique (Lazarte, 2008). L'approbation par consultation référendaire d'une nouvelle Constitution le 25 janvier 2009 en constitue sans aucun doute une étape importante. Pour comprendre les enjeux que pose cette nouvelle Constitution, il est nécessaire de regarder l'évolution politique depuis les années 1980. Cette dernière explique largement l'originalité actuelle de la situation bolivienne.

2 Les changements remontent au moins au rétablissement de l'ordre constitutionnel en 1982, après les années d'autoritarisme militaire. Les Boliviens ont alors commencé à s'interroger sur le pays qu'ils souhaitaient et par conséquent, sur la nature de l'Etat. En effet, avec l'apparition progressive - due au retour à la démocratie - d'une foule de partis politiques $^{1}$ véhiculant des idées très variées, le mode de scrutin plurinominal à un tour établi par la Constitution de 1967 ne permettait pas facilement l'émergence d'une majorité de voix en faveur d'une liste. Les présidents boliviens ont donc souvent été proclamés après le vote du Congrès. S'il s'agissait d'une procédure prévue par la Constitution, ce mode de proclamation par le Congrès fragilisait la légitimité des présidents, qui ont ainsi souvent été amenés à la démission en cours de mandat (Lavaud, 2006). Or, avec 53,7 \% des voix exprimées aux élections générales de 2005 pour la liste du Movimiento al Socialismo (MAS), son leader Evo Morales a été proclamé directement président de la République (Dory, 2006). Bien élu, il jouit d'une incontestable légitimité pour mettre en place son programme de refondation du pays. Cette idée de refondation de la Bolivie est la proposition du MAS pour remédier aux crises que le pays traverse 
depuis les années 1990, et qui ont éclaté au grand jour à partir des années 2000 avec des vagues successives de conflits sociaux (Orozco Ramírez \& al., 2006 ; Crespo \& al., 2007).

Or, ces crises qui ont profondément secoué la Bolivie sont attribuées d'une part au modèle politique de démocratie représentative que les Boliviens perçoivent comme peu participatif; et d'autre part, à un modèle néolibéral où prédomine un Etat centralisé inspiré de la Révolution française (Romero Ballivián, 2003), réfractaire à la reconnaissance des spécificités régionales et ethniques, au nom de l'appartenance à une communauté politique (Lacombe, 2007 ; Lazarte, 2008). Pendant la première moitié de la décennie 2000, les différents leaders politiques ont donc testé auprès des électeurs, lors des consultations électorales, des propositions de réponses, qui s'organisent autour de deux grandes alternatives : création d'un nouvel Etat, garant d'une juste distribution des richesses, condamnant le néocolonialisme en s'appuyant sur une base indianiste ; projet libéral, autonomiste départemental plutôt inspiré du modèle espagnol sur la base de la Constitution existante (art. 109 et 110). Ces propositions, qui interrogent toute la forme de l'Etat d'une manière plus ou moins profonde, n'ont pas cessé d'aviver d'anciennes expressions régionalistes rivales (Lavaud, 2006; Lacombe, 2007; Lazarte, 2008) en accentuant toujours plus la césure entre l'ouest et l'est du pays.

4 La partie orientale du pays, régulièrement animée de velléités d'autonomie par rapport au pouvoir central représenté dans la partie andine, occidentale (Perrier-Bruslé, 2005 ; Lacombe, 2007), s'est organisée, notamment à partir du Comité civique Pro Santa Cruz apparu dans les années 1950, pour revendiquer un champ d'action élargi face à l'Etat. Elle s'appuie pour cela sur sa réussite économique - le département de Santa Cruz produit plus du tiers de la richesse nationale -, qu'elle mâtine d'un fond d'identification culturelle d'héritage hispanique, en opposition aux régions andines du pays. Elle a entraîné dans son sillage les départements orientaux du pays (Beni, Pando, Tarija, et dans une moindre mesure Chuquisaca), constituant ce qu'on appelle communément les départements de la demi-lune ou Media Luna (Perrier-Bruslé, 2005; Lacombe, 2007; Blanchard, 2008), bastions, avec des nuances, des partis conservateurs et libéraux (Dory, 2006). Assez vastes, comptant la plus grande ville de Bolivie, Santa Cruz de la Sierra, ces départements sont cependant peu densément peuplés. Ils ne représentent qu'à peine plus de $40 \%$ de la population totale de la Bolivie au recensement de la population de 2001, et $36,4 \%$ des électeurs inscrits aux consultations référendaires du 25 janvier 2009. En outre, ces électeurs ne sont pas tous rangés au côté des partis conservateurs et libéraux. En effet, si ces départements connaissent un accroissement démographique positif, ce dernier est largement alimenté par un mouvement migratoire, essentiellement de colonisation agricole lié depuis la deuxième présidence de Hugo Banzer (1997-2001) à la distribution de terres en faveur des paysans des départements andins (Lacombe, 2007), les plus pauvres du pays, au grand dam des populations de l'est.

5 Ces problèmes de fond qu'affronte la Bolivie sont progressivement attribués par les hommes politiques à la Constitution en vigueur. Certes, des réformes de la Constitution de 1967 ont été menées en 1994 et 2004, et ont notamment débouché sur la définition d'un Etat multiethnique et pluriculturel, décentralisé à l'échelon municipal. Mais, ces réformes sont jugées insatisfaisantes par les partis. En s'appuyant sur l'interprétation selon laquelle les conflits sociaux en Bolivie sont l'expression d'une volonté de changement profond - avec des causes différentes selon les partis -, les principaux partis politiques ont promu l'idée que seule une nouvelle Constitution pourra répondre pacifiquement à cette demande. Finalement, en mars 2006, le Congrès vote une loi spéciale pour 
convoquer une Assemblée constituante (Lazarte, 2008) et la tenue d'un référendum national sur l'autonomie départementale.

6 En juillet 2006, les départements occidentaux se prononcent contre l'autonomie, alors que ceux de l'est votent largement en sa faveur (Dory, 2006). Ils ont donc l'assurance d'accéder à un statut d'autonomie, mais dans un cadre que devra définir une nouvelle Constitution. Les représentants à l'Assemblée constituante, élus durant le même scrutin, en majorité du MAS, mais pas de manière absolue, se sont donc efforcés de rédiger une Constitution destinée à limiter la portée de l'autonomie. C'est pourquoi, l'approbation ou non de la nouvelle Constitution constitue un enjeu pour les différents partis politiques. Derrière les "questions d'autonomies à enrobage régionalistes ou ethnique " (Lavaud, 2006 : 76), la nouvelle Constitution pose aussi la question de la propriété des ressources naturelles (propriété de la terre et propriété des revenus liés à l'exploitation des ressources du sous-sol), question économique sur laquelle se joue aussi la refondation du pays.

\section{Contexte bolivien pré référendum}

7 Un peu moins de quatre millions de Boliviens ${ }^{2}$ ont été consultés le 25 janvier 2009 à propos de deux mesures proposées par le pouvoir exécutif: un référendum sur la limitation de la superficie maximale de la propriété foncière ( 5000 ha ou 10000 ha), un autre sur la ratification du texte d'une nouvelle Constitution pour régir la Bolivie. Cette dernière mesure, sûrement parce qu'elle est la réforme la plus ambitieuse du Président Evo Morales (Morales, 2008), mais aussi par ses conséquences sur l'organisation du régime de l'Etat et du fonctionnement de la société, a retenu l'attention internationale.

Contrairement à l'impression de facilité donnée par son résultat, la ratification de cette nouvelle Constitution de la Bolivie s'est faite dans la douleur. La nouvelle Constitution, annoncée par Evo Morales dans son programme de candidat lors de la campagne pour l'élection présidentielle en 2005, afin de refonder la société bolivienne, a en effet été fortement instrumentalisée par les partis d'opposition (PODEMOS, PULSO, etc.) pour critiquer l'orientation de la politique du parti du Président élu et de ses principaux alliés (MAS, MSM, etc.). Aussi, pendant les deux années durant lesquelles une Assemblée constituante élue en juillet 2006 a siégé pour la rédiger, parallèlement aux deux chambres du Congrès, des troubles souvent violents, mortels parfois, ont-ils régulièrement convulsé le pays (Blanchard, 2008 ; Lazarte, 2008 ; EUEOM, 2009).

9 Les semaines précédant la consultation du 25 janvier 2009 ont donc été particulièrement tendues, avec des campagnes très agressives, souvent fallacieuses, menées par les partisans des différents camps, qui n'ont pas toujours fait la part entre fonds publics dont ils ont la gestion et fonds de campagne de leurs partis (Figure $n^{\circ} 1$ ). Pendant les 60 jours de campagne des deux référendums, l'opposition au gouvernement a aussi diffusé à Cochabamba des tracts qui tentaient de démontrer que l'approbation de la nouvelle Constitution entrainerait le chaos, en créant des pénuries d'eau, de gaz et d'électricité. 
Figure $n^{\circ} 1$ - Rassemblement en faveur de l'approbation du texte de la nouvelle Constitution

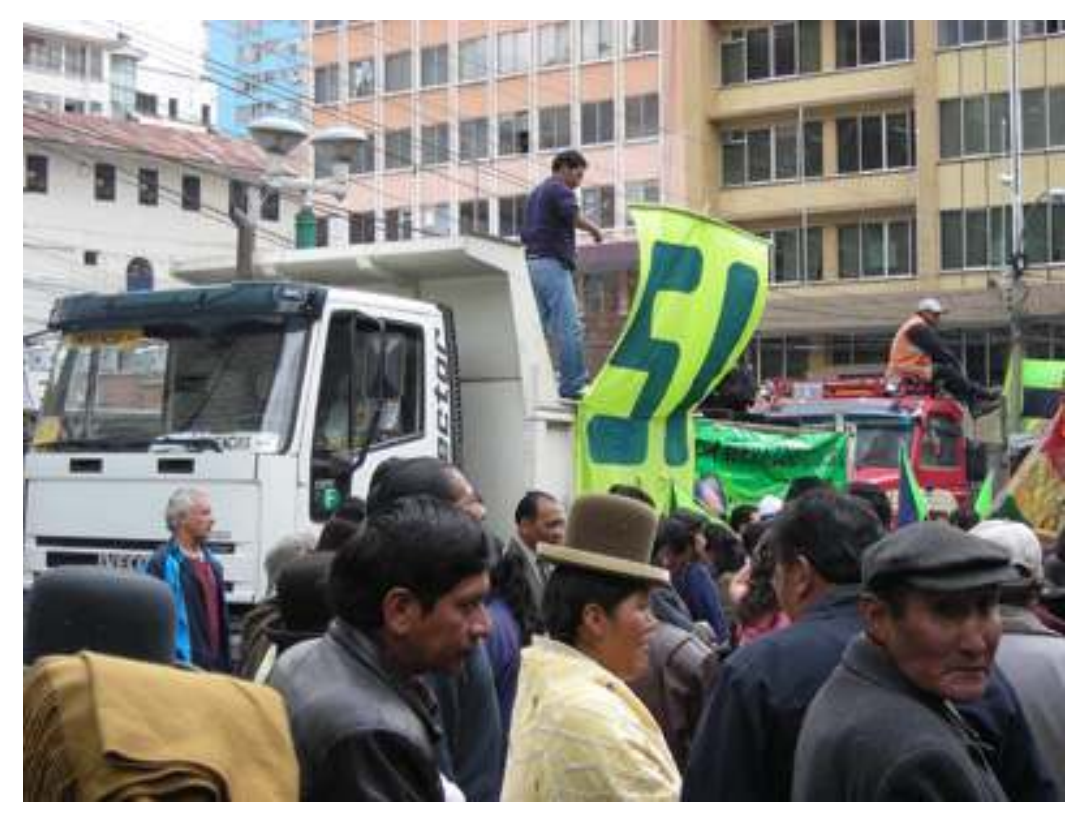

Rassemblement organisé par le Movimiento Sin Miedo (MSM), parti du maire de La Paz, allié du Movimiento Al Socialismo (MAS) d'Evo Morales. Une partie des populations qui y participent a été amenée devant la mairie par des camions du Gouvernement municipal de La Paz normalement affectés à la gestion des urgences provoquées par les risques d'origine naturelle (Emergencias) (Cliché : S. Hardy, janvier 2009).

Dans ce contexte, pour légitimer les résultats, les acteurs politiques se sont entendus sur la présence dans le pays de missions internationales pour observer la consultation (EUEOM, OEA, the Carter Center). En parfaite collaboration avec les représentants des partis politiques, les organisations civiles, la Cour nationale électorale (CNE), les forces de maintien de l'ordre, les observateurs de la mission européenne ont par exemple pu très librement, dès le 14 décembre 2008, mettre en place l'observation des consultations à partir d'une méthodologie rigoureuse et impartiale, suivant toutes les différentes étapes de la consultation (avant, pendant, après). Aussi les accusations de fraudes lancées par les opposants à Evo Morales les heures suivant les résultats, destinées à discréditer la validité de ces derniers, n'ont-elles aucun fondement. Aucune perturbation significative n'a d'ailleurs entravé le bon déroulement des deux référendums (EUEOM, 2009).

11 S'il y a des réserves à formuler, elles tiennent plutôt au fonctionnement des tables de vote par rapport à ce qu'établit le code électoral. Les dysfonctionnements observés relèvent essentiellement de la compréhension par les responsables des tables de vote, des règles du code électoral, mais sont mineurs: par exemple, le non respect de l'ordre des procédures de vote, non présence systématique à chaque table d'un représentant des forces de l'ordre. Ses dysfonctionnements ne font qu'éclairer les difficultés à faire fonctionner un système démocratique dans le pays le plus pauvre d'Amérique du Sud : les responsables des tables de vote sont des citoyens tirés au sort. Malgré les critères de sélection retenus pour leur désignation, de nombreux responsables maitrisent souvent mal la compréhension des consignes du code électoral. Ces dysfonctionnements n'influent toutefois en rien les résultats. 


\section{Les résultats à l'échelle bolivienne}

12 Le 25 janvier, le taux de participation a atteint 90,24\% des inscrits : 61,43\% des votants ont approuvé la nouvelle Constitution et $80,65 \%$ se sont exprimés en faveur de la limitation de la superficie maximale de la propriété foncière à 5000 ha (CNE, 2009). De tels résultats devraient confortablement renforcer la politique suivie par Evo Morales. Pourtant, l'analyse de ces résultats souligne les difficultés que la Bolivie traverse, et les défis qui seront encore à relever.

Le taux de participation dissimule une profonde lassitude des Boliviens à être consultés. Le code électoral bolivien oblige en effet le citoyen âgé d'au moins 18 ans à voter. Après avoir voté, il reçoit un certificat de vote, qu'il doit impérativement présenter pour réaliser des démarches de la vie quotidienne : opérations bancaires, demande de papiers d'identités, etc. Le citoyen qui n'a pas voté doit le justifier : en apportant la preuve qu'il est âgé de plus de 70 ans (il n'a alors plus l'obligation de voter) ; en prouvant qu'une force majeure l'a empêché de s'exprimer ; en s'acquittant du paiement d'une amende pour non accomplissement de ses devoirs. Dans le cas contraire, il est automatiquement rayé des listes électorales. Le taux de participation élevé reflète donc avant tout ces contraintes, lesquelles incitent fortement à voter, et non l'intérêt réel des citoyens pour l'enjeu soumis au vote.

Par ailleurs, souvent consultés par voie référendaire (le dernier avait eu lieu le 10 août 2008) ce que de nombreux auteurs nomme «la fièvre référendaire bolivienne » (Blanchard, 2008 ; Romero Ballivián, 2008, Morales, 2008), l'intérêt des Boliviens s'érode progressivement (Lazarte, 2008 : 5). Dans le cas des référendums du 25 janvier 2009, peu de citoyens ont ainsi réellement pris la peine de lire le texte, pourtant court, de la nouvelle Constitution proposée, de comprendre ses différences par rapport à celle qui était alors en vigueur. Ils sont conscients que les consultations servent avant tout à trancher des différends politiques (Blanchard, 2008), plutôt qu'à obtenir le consensus politique pour permettre au pays de progresser. Ils ont certes voté plus que lors du référendum révocatoire d'août 2008 où l'abstention avait atteint $17 \%$. Mais, le nombre d'inscrits sur les listes a aussi beaucoup diminué entre août 2008 et janvier 2009 (156 309 électeurs en moins)! La mission d'observation électorale de l'Union européenne a plusieurs fois souligné les nombreuses imperfections des listes électorales en Bolivie, et les problèmes qu'elles soulèvent ainsi pour le fonctionnement démocratique (EUEOM, 2009).

Quant aux résultats, derrière la satisfaction légitime pour Evo Morales et ses alliés d'avoir tenu une promesse du programme de campagne électorale, ils sont aussi interprétés par le leader des gauches avec une certaine inquiétude. Un référendum révocatoire avait déjà été organisé en août 2008, avec deux questions distinctes : l'une qui remettait en jeu son mandat de Président, l'autre celui des préfets des départements (Blanchard, 2008). Cette consultation l'avait plébiscité, avec un résultat de $63,1 \%$ de votants approuvant sa politique. Il avait toutefois dans le même temps permis le plébiscite des préfets des départements orientaux qui mènent la fronde contre le Président élu. Si l'on considère ces deux consultations référendaires, celle de 2008 et celle de 2009 comme des plébiscites, on remarque en un peu moins de six mois, une érosion du soutien des citoyens au Président et à sa politique - même si le soutien reste incontestable. Ce constat doit donc 
être mis en perspective, afin d'anticiper la prochaine consultation, prévue le 6 décembre 2009 : l'élection présidentielle et l'élection des représentants au Congrès.

Dès la publication des résultats du 25 janvier, l'équipe politique d'Evo Morales a d'ailleurs émis l'idée d'anticiper la prochaine échéance électorale. La ratification de la nouvelle Constitution ne préjuge en effet en rien de l'application de ses 411 articles. Pour cela, Evo Morales aura besoin d'un Congrès fort, ce que de nouvelles élections anticipées pourraient lui apporter. Mais, son actuelle réussite politique tenait en grande partie à la relative bonne marche de l'économie bolivienne depuis le début de son mandat en 2006 (Blanquer, Zagefka, 2005), qui lui a permis une politique assez généreuse de redistribution (Morales, 2008), notamment vers les plus pauvres, très largement majoritaires en Bolivie (Romero Ballivián, 2008). Or, les mauvaises nouvelles économiques ne font que s'accumuler depuis plusieurs mois, reflet de la situation économique mondiale : baisse de la demande en gaz bolivien des partenaires brésiliens et argentins, rapide hausse de l'inflation ( $12 \%$ en 2007 alors que la Bolivie était habituée ces dernières années à un taux inférieur à $5 \%$ ). Elles commencent à affecter le contentement des citoyens, surtout dans les villes, même dans les départements favorables au MAS. Dans ce contexte, le soutien de la population dans sa majorité pourrait fortement se dégrader, renforçant par la même occasion la polarisation politique entre une partie occidentale du pays qui est plutôt favorable à Evo Morales et à sa politique de refondation de la Bolivie, et une partie orientale qui lui est de plus en plus hostile.

\section{Lecture des résultats à l'échelle provinciale}

17 La ratification de la nouvelle Constitution ne signifie pas la fin des conflits intérieurs. La distribution spatiale des résultats des consultations du 25 janvier montrent ainsi une nette opposition entre les départements de l'est du pays (Tarija, Santa Cruz, Beni, Pando), où l'opposition est active et domine la scène politique (Dory, 2006; Lavaud, 2006; Lacombe, 2007), et où la population a exprimé son refus du texte de la nouvelle Constitution, d'avec ceux de l'Altiplano et des vallées centrales (La Paz, Cochabamba, Oruro, Potosí, Chuquisaca) où la population l'a approuvé (figure n ${ }^{\circ} 2$ et figure $\mathrm{n}^{\circ} 3$ ).

Figure $n^{\circ} 2$ - Distribution départementale des voix en faveur du oui au texte de la nouvelle Constitution. 

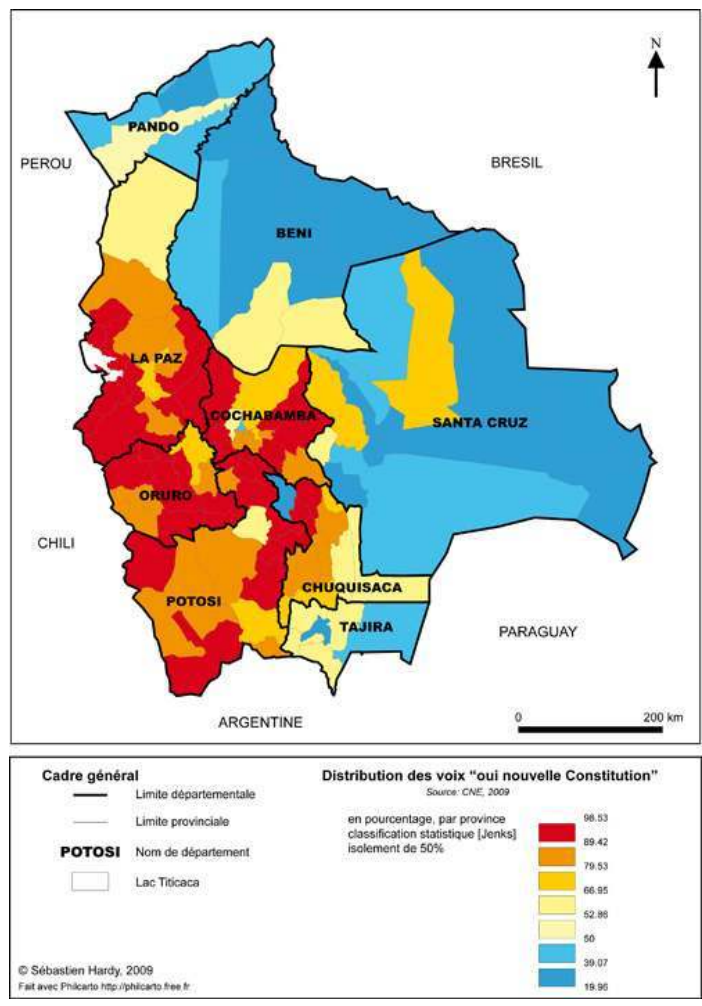

Figure $n^{\circ} 3$ - Distribution départementale des voix en faveur de la limitation de la superficie maximale de la propriété foncière à 5000 ha

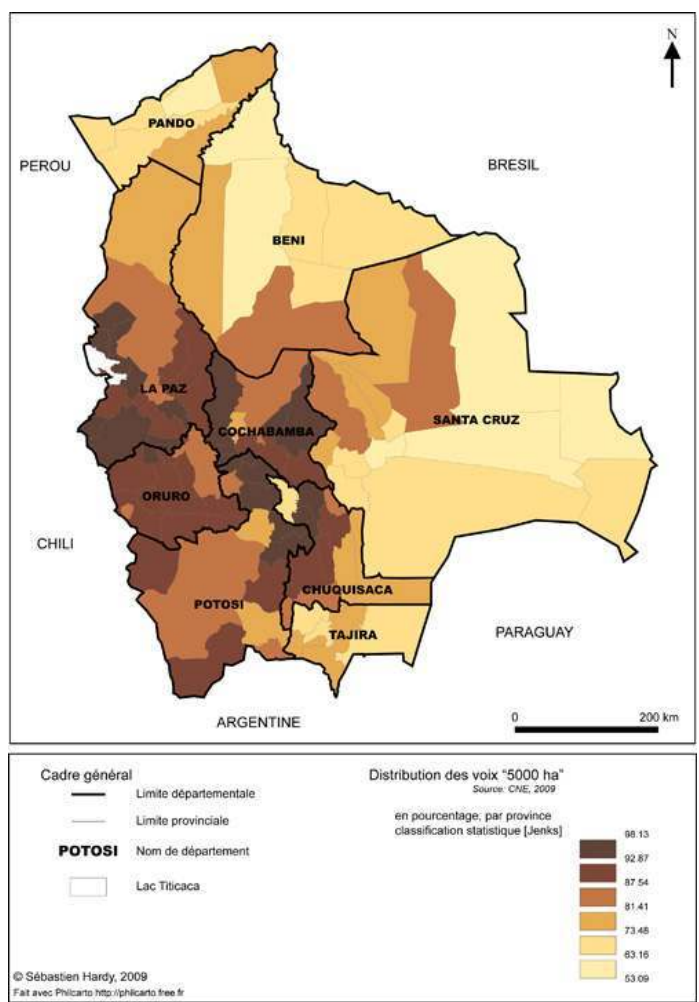

19 En changeant d'échelle, l'observation des résultats dans les provinces permet de souligner une polarisation toujours plus forte entre les villes et les campagnes, de nombreuses villes préfectures de département ayant exprimé un vote moins favorable pour la Constitution 
et pour la limitation à 5000 ha de la propriété foncière, quand les campagnes ont exprimé un désir de poursuite des changements. Par exemple, dans la province de Oropeza, département de Chuquisaca, qui abrite l'agglomération de Sucre, on observe que le refus de la nouvelle Constitution est fort: le oui à la Constitution n'atteint que $33,78 \%$. En revanche, les autres provinces du département, rurales, l'ont accepté, mais parfois de peu. Par conséquent, le oui l'emporte de justesse dans le département de Chuquisaca, avec $51,54 \%$ des votes en faveur sur l'ensemble de ce département: le poids démographique de l'agglomération de Sucre par rapport au reste du territoire départemental explique ce résultat. Quand on observe les départements bastions politiques, ceux du MAS et ceux de l'opposition, les résultats à l'échelle des provinces rendent une image plus contrastée.

Dans le département de Cochabamba, on observe un refus dans l'agglomération de Cochabamba du texte de la nouvelle Constitution ( $47 \%$ de oui), quand les autres provinces, dont celle du Chaparé, un bastion du MAS, l'acceptent. A l'échelle des autres départements de l'Altiplano, on observe à nouveau que les villes n'ont pas voté massivement pour cette nouvelle Constitution : La Paz l'accepte à $60,20 \%$, Oruro à $64,12 \%$ et Potosí à $58,90 \%$. Les résultats dans ces trois villes contrastent fortement avec les provinces rurales de leurs alentours.

21 Dans les bastions de l'opposition, on observe aussi les contrastes entre villes et campagnes. Quelques zones rurales ont nettement voté oui, quand les municipalités urbaines ont souvent refusé la nouvelle Constitution. La progression de la colonisation agricole dans ces départements par des populations qui proviennent des parties occidentales du pays depuis l'arrivée au pouvoir d'Evo Morales explique que certaines provinces y acceptent la nouvelle Constitution. Dans les départements orientaux, c'est donc essentiellement le poids démographique des villes, où l'opposition est forte, qui font basculer le vote vers le refus du texte de la nouvelle Constitution.

Les dichotomies Ouest/Est en Bolivie bien connues (Alenda, 2003 ; Perrier-Bruslé, 2006 ; Dory, 2006; Lavaud, 2006; Lacombe, 2007; Morales, 2008; Romero Ballivián, 2008) expliquent aussi pourquoi la limitation de la superficie maximale de la propriété foncière à 5000 ha est moins acceptée par les électeurs des départements de l'est du pays (figure $\mathrm{n}^{\circ}$ 3) qu'à l'ouest. La réforme agraire de 1953 a en effet été peu appliquée dans l'Est, où dans les années 1960 l'Etat a en outre distribué de grandes étendues de terres à un petit groupe de propriétaires, favorisant la formation de latifundia (Blanchard, 2008). Par ailleurs, les décisions gouvernementales pour faire face à la dégradation des indicateurs économiques ces derniers mois ont attisé le mécontentement des grands propriétaires producteursexportateurs des départements orientaux du pays. Pour ralentir l'inflation, le gouvernement a interdit la vente à l'extérieur de certains produits agricoles, les frappant en premier lieu (Morales, 2008).

En revanche, la forte croissance démographique a produit un extrême fractionnement des parcelles cultivables dans l'Ouest. Cette situation peut facilement expliquer que ces départements occidentaux aient voté à plus de $80 \%$ pour la limitation de la superficie maximale de la propriété foncière à 5000 ha. La colonisation agricole dans les départements orientaux par des paysans souvent sans terre provenant de l'Ouest (Morales, 2008), qui ont déjà bénéficié de redistribution de terres ou même qui ont commencé à occuper quelques grandes propriétés, explique aussi que certaines provinces orientales aient voté fortement pour la restriction de la propriété foncière à 5000 ha. Globalement, les paysans attendent du MAS une nouvelle réforme agraire, comme celle 
qui leur avait été fortement favorable en 1953, et qui depuis fait apparaître ces électeurs, originaire des départements andins, comme une réserve de voix pour le MAS.

En refusant le texte de la nouvelle Constitution (figure ${ }^{\circ} 2$ ), les opposants à Evo Morales contredisent aussi une de leurs revendications : un degré plus élevé d'autonomie. Cette revendication déjà ancienne d'autonomie administrative et politique des départements (Alenda, 2003), clairement rappelée lors du référendum de 2006, a été intégrée dans le texte de la nouvelle Constitution. Dans sa troisième partie, elle prévoit en effet la possibilité de l'autonomie, sur des bases qui ne se fondent pas sur une continuité territoriale (par exemple deux municipalités non contiguës), mais sur la volonté d'association. La définition, qui à l'heure actuelle n'existe pas, des champs de l'autonomie sera donc un enjeu important des prochains mois.

Or, les départements de Santa Cruz et Tarija sont sans conteste les moteurs économiques du pays grâce aux ressources tirées respectivement de l'agriculture exportatrice et des hydrocarbures, à partir desquelles le gouvernement finance sa politique de redistribution. Ils exploiteront donc sûrement toutes les voies possibles pour définir une autonomie très poussée, surtout dans le domaine économique. En agissant ainsi, ils pourraient par la même occasion saper un des piliers qui rendent Evo Morales crédible dans son action pour refonder la Bolivie. Au regard de ce contexte de l'autonomie, on peut légitimement se demander comment l'article un de la Constitution, qui crée un Etat unitaire, social, de droit plurinational, communautaire pourra s'appliquer. Il est surtout difficile d'imaginer que des tensions n'en découleront pas.

Toutes ces données augurent donc encore de troubles prévisibles en Bolivie au cours des prochains mois de l'année 2009.

\section{BIBLIOGRAPHIE}

Alenda S., 2003, « Bolivie, les limites de la consolidation institutionnelle : l'érosion du pacte démocratique ", Problèmes d'Amérique latine, n 49, p. 71-99.

Blanquer J-M., Zagefka P., 2005, Amérique latine, Paris, La documentation française, 204 p.

Blanchard S., 2008, « Bolivie, de l'autonomie à l'éclatement? », EchoGéo, Sur le vif 2008, [En ligne] URL : http://echogeo.revues.org/index5593.html.

$\mathrm{CNE}$, « Resultados referéndum nacional Constituyente 2009 », Documento de información pública, $\mathrm{n}$ $\circ$, $24 \mathrm{p}$.

Crespo C., Spronk S. (coord.), 2007, Después de las guerras del agua, movilizaciones políticas, La Paz, Plural Editores, $245 \mathrm{p}$.

Dory D., 2006, « Polarisation politique et fractures territoriales en Bolivie », Hérodote, $\mathrm{n}^{\circ} 123$, p. 82-87.

EUEOM, 2009, Preliminary statement: Constitutional referendum, 25 January 2009, La Paz, EUEOM, 13 p., [En ligne] URL : http://www.eueombolivia.org 
Lacombe Z., 2007, « Identité et politique à Santa Cruz, Bolivie. Articulations au service d'un projet politique régionaliste », Outre-Terre, 2007/1, pp. 273-294.

Lavaud J-P., 2006, « Bolivie : vers l'anarchie segmentaire ? L'« ethnicisation » de la vie politique », Hérodote, $\mathrm{n}^{\circ} 123$, p. 62-81.

Lazarte J., 2008, « La Asamblea Constituyente de Bolivia: de la oportunidad a la amenaza », Nuevo Mundo Mundos Nuevos, Cuestiones del tiempo presente, 2008, [En ligne] URL http:// nuevomundo.revues.org/index42663.html

Morales J., 2008, «L'économie politique du populisme bolivien du XXI ${ }^{\mathrm{e}}$ siècle », Problèmes d'Amérique latine, $\mathrm{n}^{\circ} 69$, p. 29-48.

Orozco Ramírez S., García Linera Á., Stefanoni P. (eds.), 2006, No somos juguete de nadie...”. Análisis de la relación de movimientos sociales, recursos naturales, Estado y descentralización, La Paz, Plural Editores, $419 \mathrm{p}$.

Perrier-Bruslé L, 2005, La dernière frontière. Loin des Andes, trop près du Brésil. La frontière orientale et la construction du territoire en Bolivie, thèse de doctorat de géographie, Paris, Université Paris 1 , $734 \mathrm{p}$.

Romero Ballivián S., 2003, Geografía electoral de Bolivia, tercera edición, La Paz, Fundemos, 510 p.

Romero Ballivián S., 2008, « Suffrage universel et démocratie en Bolivie : regard sur un demisiècle ", Problèmes d'Amérique latine, $\mathrm{n}^{\circ}$ 69, p. 11-28.

\section{NOTES}

1. On peut notamment citer les grands partis actuels : PODEMOS : Poder Democrático Social = Pouvoir démocratique social ; MAS : Movimiento Al Socialismo = Mouvement vers le Socialisme ; MSM : Movimiento Sin Miedo = Mouvement sans peur.

2. Le nombre d'électeurs inscrits sur les listes pour cette consultation a été arrêté à 3891 316. La Bolivie compte un peu plus de 9 millions d'habitants.

\section{RÉSUMÉS}

La troisième consultation référendaire organisée légalement sous le mandat du Président Evo Morales (2006-2010) a eu lieu le 25 janvier 2009. L'enjeu de cette consultation portait sur l'approbation du texte d'une nouvelle Constitution pour la Bolivie, ainsi que sur la limitation de la superficie de la propriété foncière. Les résultats pris globalement laissent penser que les propositions $\mathrm{du}$ Président satisfont les Boliviens. Pourtant, en changeant l'échelle d'interprétation des résultats, on retrouve les classiques dissensions qui polarisent depuis longtemps, et toujours plus, le pays.

The third legally organized referendum under the mandate of President Evo Morales (2006-2010) took place on January 25,2009 . What is at stake in this consultation is the approval of the text of a new Constitution for Bolivia, and the limitation of the area of the land ownership. The results taken as a whole lead one to believe that the proposals of the President satisfy Bolivians. 
However, by looking at the bigger picture, one finds the traditional dissensions which have polarized the country for a long time, and continue to do so.

La tercera consulta por referéndum organizada legalmente bajo el mandato del Presidente Evo Morales (2006-2010) tuvo lugar el 25 de enero de 2009. El desafío de esta consulta se refería a la aprobación del texto de una nueva Constitución para Bolivia, y a la limitación de la superficie de la propiedad de la tierra. Globalmente, los resultados dejan pensar que las propuestas del Presidente satisfacen a los Bolivianos. Sin embargo, al cambiar la escala de interpretación de los resultados, se encuentran las clásicas disensiones que polarizan desde hace tiempo, y siempre más, el país.

\section{INDEX}

Palabras claves : Bolivia, referéndum, regionalismo, tenencia de la tierra, constitución

Keywords : Bolivia, referendum, regionalism, land ownership, constitution

Mots-clés : Bolivie, référendum, régionalisme, foncier, constitution

\section{AUTEUR}

\section{SÉBASTIEN HARDY}

Sébastien Hardy (sebastien.hardy@ird.fr) est chargé de recherche à Institut de Recherche pour le Développement, UR 029, programme PACIVUR. Il a publié récemment :

- Hardy S., (2009), « Explorer la construction de la résilience. Expériences de recherche à La Paz », Peltier A., Becerra S. (dir.), Vulnérabilités sociétales, risques et environnement, Editions L'Harmattan, Paris.

- Hardy. S, (2008), « Ciudad Sandino, marge urbaine en périphérie de Managua ?», Annales de géographie, 662, pp. 85-103.

- Hardy S., (2007), « Choluteca, ville hondurienne du système Monde? », Cybergeo, Espace, Société, Territoire, article 379, URL: http://www.cybergeo.eu/index7242.html. 